\title{
Chaos in Discrete Structured Population Models*
}

\author{
Eduardo Liz L $^{\dagger}$ and Alfonso Ruiz-Herrera ${ }^{\ddagger}$
}

\begin{abstract}
We prove analytically the existence of chaotic dynamics in some classical discrete-time age-structured population models. Our approach allows us to estimate the sensitive dependence on the initial conditions, regions of initial data with chaotic behavior, and explicit ranges of parameters for which the considered models display chaos. These properties have important implications for evaluating the influence of a chaotic regime on the predictions based on mathematical models. We illustrate through particular examples how to apply our results.
\end{abstract}

Key words. chaotic dynamics, coin-tossing, structured population models, Clark equation, LPA model

AMS subject classifications. 37D45, 37N25, 39A33, 92D25

DOI. $10.1137 / 120868980$

1. Introduction. Understanding chaos is an important aim in many disciplines. May $[24,25]$ has already pointed out the possibility that simple deterministic models will display complex behavior, and the importance of chaos in ecology. The main implication of erratic fluctuations typical of a chaotic system is that long-time predictions are not possible; as emphasized by Hastings et al. [15], the characteristic of chaos that better represents this difficulty is a sensitive dependence on initial conditions. Besides this property, there are other important features in a chaotic system. Intuitively, and according to Smale's expository article [28], a chaotic phenomenon occurs if it is possible to reproduce, within the system and varying the initial conditions, all the possible outcomes of a coin-tossing experiment (see Definition 3.1). This notion has strong implications such as sensitive dependence on the initial conditions and the existence of aperiodic orbits and infinitely many periodic points.

Although analytic proofs of chaos are now available for some one-dimensional maps usually employed in population dynamics (see, e.g., Thunberg's survey [30] and references therein), it is hard to find evidence of chaos in ecology. The main reason is the difficulty in manipulating and experimenting with ecological systems. A remarkable exception is the work of Cushing and coworkers $[7,8,10,11]$. They derived a structured population model, known as the LPA (larvae, pupae, and adults) model, to study the growth of laboratory populations of flour beetles of the genus Tribolium, and their experiments confirmed chaotic behavior predicted by the model. Other age-structured population models in which chaos has been recently explored

\footnotetext{
${ }^{*}$ Received by the editors March 6, 2012; accepted for publication (in revised form) by L.-S. Young June 29, 2012; published electronically October 4, 2012.

http://www.siam.org/journals/siads/11-4/86898.html

${ }^{\dagger}$ Departamento de Matemática Aplicada II, E. I. Telecomunicación, Universidad de Vigo, 36310 Vigo, Spain (eliz@dma.uvigo.es). This author's work was supported in part by the Spanish Government and FEDER, grant MTM2010-14837.

¥Departamento de Matemática Aplicada, Facultad de Ciencias, Universidad de Granada, 18071 Granada, Spain (alfonsoruiz@ugr.es). This author's work was supported in part by the Spanish Government, project MTM201123652.
} 
are Clark's equation $[3,16]$ and a class of density-dependent Leslie models [14, 19, 32, 33, 35].

The aim of this paper is twofold. On the one hand, we provide analytic proofs of existence of chaotic dynamics in some systems of difference equations, and we apply our results to the aforementioned structured population models. From a biological point of view, our strategy consists of introducing chaos in a specific age group and then studying its influence in the full system; from a mathematical perspective, we derive criteria of chaotic dynamics in higher-dimensional systems using a generalization of some elementary notions of chaos in one dimension. On the other hand, an important purpose of this work is to interpret some natural properties of complex dynamics in ecological models. Specifically, we stress the estimation of the sensitive dependence on the initial conditions and the location of regions of both the phase space and the set of parameters where the system displays chaos. These two properties have important practical implications for evaluating the influence of a chaotic regime on predictions based on mathematical models.

The organization of the paper is as follows. In section 2, we review the specific population models in which we focus our study; in section 3, we introduce the notion of chaotic dynamics that we use and derive some results about chaos for continuous higher-dimensional maps. Sections 4 and 5 contain our main applications for proving chaos in the structured models introduced in section 2; some computations are included in two appendices. Finally, in section 6 , we discuss the main conclusions of this paper.

2. Structured population models. Mathematical formulation of discrete models leads us to consider difference equations or matrix models, which are nonlinear if changes in response to population density are taken into account. We focus our study on discrete-time agestructured models for a single population; that is to say, the model describes the distribution of individuals among the possible categories of important age differences. The monographs $[4,9,31]$ are good sources both for theory and applications of structured population models.

Perhaps the simplest formulation that combines age structure and density-dependent recruitment has the form

$$
x_{n}=\alpha x_{n-1}+(1-\alpha) f\left(x_{n-k}\right),
$$

which is commonly known as the Clark model $[3,5]$ and has been employed in fishery models (see [26] and its references). For $k=1,(2.1)$ is a first-order difference equation that provides a simple way to allow for certain survivorship of adults from one reproduction period to the next. This one-dimensional model was formulated by Clark [6]; see also [29, section 9.2]. For recent work involving this equation, including chaotic dynamics, we refer the reader to $[21,23,36]$.

For $k \geq 2$, we do not know any analytic proof of chaos in (2.1). Botsford [3] has already reported some numerical results suggesting chaotic behavior in (2.1) with the Ricker map $f(x)=x e^{r(1-x)}, r>0$. For a more detailed study, including numerical computations of Lyapunov exponents for $k=3$, we refer to [16].

Another simple structured model for which existence of chaos has been explored is a density-dependent Leslie population model with two age classes,

$$
\left\{\begin{array}{l}
x_{n+1}=\left(a_{1} x_{n}+a_{2} y_{n}\right) \exp \left(-\lambda_{1} x_{n}-\lambda_{2} y_{n}\right), \\
y_{n+1}=b x_{n},
\end{array}\right.
$$


where $a_{1}, a_{2}, \lambda_{1}, \lambda_{2}$ are positive parameters and $\left.\left.b \in\right] 0,1\right]$.

Guckenheimer, Oster, and Ipaktchi [14] considered the case $a_{1}=a_{2}=a, \lambda_{1}=\lambda_{2}=0.1$, $b=1$ to show numerically a route to chaos as $a$ is increased. See also [32, 35] for a detailed study of stability and bifurcations. Recently, Ugarcovici and Weiss [33] proved the existence of a set of parameters for which system (2.2) admits a chaotic attractor.

As mentioned before, a study to predict the population dynamics of the flour beetle Tribolium published by Cushing et al. in a series of papers (see [11] and references therein) provides a structured population model that became a paradigm for chaos in ecology. The model distinguishes three stages (larvae, pupae, and adults) and is referred to as the LPA model:

$$
\left\{\begin{array}{l}
L_{n+1}=b A_{n} \exp \left(-c_{e l} L_{n}-c_{e a} A_{n}\right), \\
P_{n+1}=\left(1-\mu_{l}\right) L_{n}, \\
A_{n+1}=P_{n} \exp \left(-c_{p a} A_{n}\right)+\left(1-\mu_{a}\right) A_{n} .
\end{array}\right.
$$

All coefficients are nonnegative, and $\mu_{l}<1, \mu_{a} \leq 1$. See, e.g., [12] for a biological interpretation of the involved parameters and many properties of this system. A route to chaos numerically predicted by the LPA model (2.3) was confirmed by some laboratory experiments. However, as stated in [11], no proof of chaotic dynamics is available.

3. Background on chaotic dynamics. In this section we give the main tools and definitions that we use in this paper. We understand chaos in the sense of the following definition.

Definition 3.1. Consider $(X, d)$ a metric space. We say that a continuous map $\psi: X \rightarrow X$ induces chaotic dynamics on two symbols if there exist two disjoint compact sets $\mathcal{K}_{0}, \mathcal{K}_{1} \subset X$ such that, for each two-sided sequence $\left(s_{i}\right)_{i \in \mathbb{Z}} \in\{0,1\}^{\mathbb{Z}}$, there exists a corresponding sequence $\left(\omega_{i}\right)_{i \in \mathbb{Z}} \in\left(\mathcal{K}_{0} \cup \mathcal{K}_{1}\right)^{\mathbb{Z}}$ such that

$$
\omega_{i} \in \mathcal{K}_{s_{i}} \quad \text { and } \quad \omega_{i+1}=\psi\left(\omega_{i}\right) \quad \text { for all } i \in \mathbb{Z},
$$

and, whenever $\left(s_{i}\right)_{i \in \mathbb{Z}}$ is a $k$-periodic sequence (that is, $s_{i+k}=s_{i}$ for all $i \in \mathbb{Z}$ ) for some $k \geq 1$, there exists a $k$-periodic sequence $\left(\omega_{i}\right)_{i \in \mathbb{Z}} \in\left(\mathcal{K}_{0} \cup \mathcal{K}_{1}\right)^{\mathbb{Z}}$ satisfying (3.1).

Definition 3.1 guarantees natural properties of complex dynamics such as sensitive dependence on the initial conditions or the presence of an invariant set $\Lambda$ being transitive and semiconjugate with the Bernoulli shift. See Theorem 2.2 in [27] for a list of properties of the map $\psi$ that hold if the conditions of Definition 3.1 are satisfied. We note that a map that is chaotic according to Definition 3.1 is also chaotic in the sense of Block and Coppel and in the sense of coin-tossing; we refer the reader to [18] and [1, Remarks 3.2] for precise definitions and further comments. Moreover, our definition of chaos ensures the existence of periodic points of any period $n \in \mathbb{N}$, in contrast with other definitions (see, e.g., [1, 2, 18]).

Our primary purpose is to obtain some criteria for detecting chaos analytically. To this end, we use the method developed by Zgliczyński and Gidea in [37] (see also [38]). Definitions 3.2 and 3.3 below correspond to Definitions 2 and 3 in [37]. We employ the usual maximum norm in $\mathbb{R}^{n}$,

$$
\left\|\left(x_{1}, x_{2}, \ldots, x_{n}\right)\right\|=\max \left\{\left|x_{i}\right|: i=1,2, \ldots, n\right\},
$$

and use the notation $J_{n}=[-1,1]^{n}$ for the closed ball of radius 1 centered at $0 \in \mathbb{R}^{n}$.

Definition 3.2. An h-set is a quadruple consisting of 
- a compact subset $N$ of $\mathbb{R}^{n}$,

- a pair of numbers $u=u(N), s=s(N) \in\{0,1,2, \ldots\}$, with $u+s=n$,

- a homeomorphism $c_{N}: \mathbb{R}^{n} \longrightarrow \mathbb{R}^{n}$ such that $c_{N}(N)=J_{n}$.

In this setting, we employ the notation

$$
\begin{aligned}
& N_{c}^{-}=\partial J_{u} \times J_{s}, \\
& N_{c}^{+}=J_{u} \times \partial J_{s} .
\end{aligned}
$$

As mentioned in [37], the numbers $u(N)$ and $s(N)$ stand for the dimensions of nominally unstable and stable directions, respectively. Notice that if $u(N)=0$, then $N_{c}^{-}=\emptyset$, and if $s(N)=0$, then $N_{c}^{+}=\emptyset$.

Definition 3.3. Assume that $N$ and $M$ are h-sets such that $u(N)=u(M)=u$ and $s(N)=$ $s(M)=s$. Let $f: N \longrightarrow \mathbb{R}^{n}$ be a continuous map, and define $f_{c}=c_{M} \circ f \circ c_{N}^{-1}: J_{n} \longrightarrow \mathbb{R}^{n}$. We say that $N$ f-covers $M$, and write it as

$$
N \stackrel{f}{\Longrightarrow} M,
$$

if the following conditions are satisfied:

1. There exists a continuous homotopy $H:[0,1] \times J_{n} \longrightarrow \mathbb{R}^{n}$ such that the following conditions hold true:

$$
\begin{aligned}
& H_{0}=f_{c}, \\
& H\left([0,1], N_{c}^{-}\right) \cap J_{n}=\emptyset, \\
& H\left([0,1], J_{n}\right) \cap M_{c}^{+}=\emptyset .
\end{aligned}
$$

2. There exists a linear map $A: \mathbb{R}^{u} \longrightarrow \mathbb{R}^{u}$ such that $H_{1}(p, q)=(A p, 0)$ for $p \in J_{u}$ and $q \in J_{s}$, and $A\left(\partial J_{u}\right) \subset \mathbb{R}^{u} \backslash J_{u}$.

Next we link these concepts with existence of chaotic dynamics.

Theorem 3.4. Let $F: \mathcal{D} \subset \mathbb{R}^{n} \longrightarrow \mathbb{R}^{n}$ be a continuous map, and assume that there exist two disjoint h-sets $N_{0}$ and $N_{1}$ such that

$$
N_{i} \stackrel{F}{\Longrightarrow} N_{j}
$$

for all $i, j=0,1$. Then $F$ induces chaotic dynamics on two symbols (with compact sets $\mathcal{K}_{0}=N_{0}$ and $\left.\mathcal{K}_{1}=N_{1}\right)$.

Proof. Let us first take a sequence $s=\left(s_{i}\right) \in\{0,1\}^{\mathbb{Z}}$ so that $s_{i+k}=s_{i}$ for all $i \in \mathbb{Z}$. In this case, it follows from Theorem 4 in [37] that there exists $x \in N_{0} \cup N_{1}$ so that

$$
F^{k}(x)=x \quad \text { and } \quad F^{i}(x) \in N_{s_{i}} \quad \text { for all } i \in \mathbb{Z} .
$$

Now we prove the assertion of the theorem. Fix an arbitrary sequence $s=\left(s_{j}\right)_{j \in \mathbb{Z}}$, and, for each $i \in \mathbb{N}$, define the compact set

$$
\Gamma_{i}=\left\{\omega \in N_{s_{0}}: F^{j}(\omega) \in N_{s_{j}} \text { for all } 1 \leq j \leq i\right\} .
$$


Clearly $\Gamma_{i+1} \subset \Gamma_{i}$ and, by the previous step, $\Gamma_{i} \neq \emptyset$ for all $i \in \mathbb{N}$. Indeed, it is sufficient to consider the $(i+1)$-periodic sequence that matches $s$ for $j=0,1, \ldots, i$. Hence,

$$
\bigcap_{i=1}^{\infty} \Gamma_{i} \neq \emptyset .
$$

To conclude the proof of the theorem, we notice that a standard diagonal argument enables us to extend the result to bi-infinite sequences; see [17, Theorem 2.2].

The following result provides us with an elementary and effective method for estimating the sensitive dependence in a chaotic regime when the conditions of Theorem 3.4 hold.

Proposition 3.5. Take $N_{0}, N_{1}$, and $F: \mathcal{D} \subset \mathbb{R}^{n} \longrightarrow \mathbb{R}^{n}$ as in the statement of Theorem 3.4, and denote $d=\operatorname{dist}\left(N_{0}, N_{1}\right)>0$. For $\varepsilon>0$, we define

$$
S_{\varepsilon}=\max \left\{n \in \mathbb{N}: N_{0} \text { contains } n \text { disjoint balls of diameter } \varepsilon\right\}
$$

and

$$
N^{*}=1+\left\lceil\frac{\ln S_{\varepsilon}}{\ln 2}\right\rceil,
$$

where, for $x \in \mathbb{R},\lceil x\rceil$ denotes the ceiling of $x$, that is, the smallest integer not less than $x$.

Then, there are two points $x_{0}, y_{0}$ satisfying that

- $x_{0}, y_{0} \in N_{0}$,

- $\left\|x_{0}-y_{0}\right\|<\varepsilon$,

- $\max _{0 \leq j \leq N^{*}}\left\{\left\|F^{j}\left(x_{0}\right)-F^{j}\left(y_{0}\right)\right\|\right\}>d$.

Proof. Fix $\varepsilon>0$. Given $\tau=\left(\tau_{1}, \ldots, \tau_{N^{*}}\right)$ with $\tau_{i} \in\{0,1\}$, we define

$$
\Gamma_{\tau}=\left\{x \in N_{0}: F^{j}(x) \in N_{\tau_{j}} \text { for all } 1 \leq j \leq N^{*}\right\} .
$$

In this way, we can construct $2^{N^{*}}$ disjoint nonempty compact sets contained in $N_{0}$. Since, by definition, $S_{\varepsilon}<2^{N^{*}}$, there are two different $N^{*}$-tuples, $\tau^{\prime}, \tau^{\prime \prime}$ so that

$$
\operatorname{dist}\left(\Gamma_{\tau^{\prime}}, \Gamma_{\tau^{\prime \prime}}\right)<\varepsilon \text {. }
$$

Hence, we can choose two points $x_{0} \in \Gamma_{\tau^{\prime}}$ and $y_{0} \in \Gamma_{\tau^{\prime \prime}}$ so that $\left\|x_{0}-y_{0}\right\|<\varepsilon$.

Since $\tau^{\prime} \neq \tau^{\prime \prime}$, there exists at least one index $j_{0} \leq N^{*}$ such that $\tau_{j_{0}}^{\prime} \neq \tau_{j_{0}}^{\prime \prime}$. By the definition of $\Gamma_{\tau^{\prime}}$ and $\Gamma_{\tau^{\prime \prime}}$, it follows that

$$
F^{j_{0}}\left(x_{0}\right) \in N_{\tau_{j_{0}}^{\prime}}, \quad F^{j_{0}}\left(y_{0}\right) \in N_{\tau_{j_{0}}^{\prime \prime}}
$$

and therefore $\left\|F^{j_{0}}\left(x_{0}\right)-F^{j_{0}}\left(y_{0}\right)\right\| \geq \operatorname{dist}\left(N_{0}, N_{1}\right)=d$, as we wanted to prove.

4. Chaotic dynamics in models (2.1) and (2.3). In this section we illustrate how to apply the previous results to systems (2.1) and (2.3). For these models, we obtain analytically chaotic regimes where the map of the system has only unstable directions in the sense of Definition 3.2. To this aim, we need to introduce the following notion of turbulence, which is more restrictive than the usual one [2, Chapter II]. 
Definition 4.1. Let $I$ be a real interval, and $g: I \longrightarrow I$ a continuous map. We say that $g$ is $\delta$-strictly turbulent if there exist four constants $\beta_{0}<\beta_{1}<\gamma_{0}<\gamma_{1}$ and $\delta>0$ so that

$$
\begin{gathered}
g\left(\beta_{0}\right)<\beta_{0}-\delta<\gamma_{1}+\delta<g\left(\beta_{1}\right), \\
g\left(\gamma_{1}\right)<\beta_{0}-\delta<\gamma_{1}+\delta<g\left(\gamma_{0}\right) .
\end{gathered}
$$

We first consider the Clark model (2.1). There is a one-to-one correspondence between the solutions of (2.1) and the orbits of the map $F_{\alpha}: \mathbb{R}^{k} \rightarrow \mathbb{R}^{k}$ defined by

$$
F_{\alpha}\left(x_{1}, \ldots, x_{k}\right)=\left(x_{2}, \ldots, x_{k}, \alpha x_{k}+(1-\alpha) f\left(x_{1}\right)\right) .
$$

For this map we get the following result.

Theorem 4.2. Assume that $f$ is $\delta$-strictly turbulent with parameters $\beta_{0}<\beta_{1}<\gamma_{0}<\gamma_{1}$. Then there exists $\alpha_{0}>0$ so that, for all $0 \leq \alpha \leq \alpha_{0}, F_{\alpha}^{k}$ induces chaotic dynamics on two symbols relative to $N_{0}=\left[\beta_{0}, \beta_{1}\right]^{k}$ and $N_{1}=\left[\gamma_{0}, \gamma_{1}\right]^{k}$.

Proof. It follows from the expression of $F_{\alpha}$ that, for $\alpha=0$,

$$
F_{0}^{k}\left(x_{1}, \ldots, x_{k}\right)=\left(f\left(x_{1}\right), \ldots, f\left(x_{k}\right)\right) .
$$

Using the continuity of $F_{\alpha}$ with respect to $\alpha$, we obtain that there is a constant $\alpha_{0}>0$ so that for all $i=1, \ldots, k,\left(x_{1}, \ldots, x_{i-1}, x_{i+1}, \ldots, x_{k}\right) \in\left[\beta_{0}, \beta_{1}\right]^{k-1}$, and $\alpha \leq \alpha_{0}$,

$$
\begin{aligned}
& \left|\left(F_{\alpha}^{k}\right)_{i}\left(x_{1}, \ldots, x_{i-1}, \beta_{0}, x_{1+i}, \ldots, x_{k}\right)-f\left(\beta_{0}\right)\right|<\delta, \\
& \left|\left(F_{\alpha}^{k}\right)_{i}\left(x_{1}, \ldots, x_{i-1}, \beta_{1}, x_{1+i}, \ldots, x_{k}\right)-f\left(\beta_{1}\right)\right|<\delta .
\end{aligned}
$$

Analogously, for all $i=1, \ldots, k$ and $\left(x_{1}, \ldots, x_{i-1}, x_{i+1}, \ldots, x_{k}\right) \in\left[\gamma_{0}, \gamma_{1}\right]^{k-1}$,

$$
\begin{aligned}
& \left|\left(F_{\alpha}^{k}\right)_{i}\left(x_{1}, \ldots, x_{i-1}, \gamma_{0}, x_{1+i}, \ldots, x_{k}\right)-f\left(\gamma_{0}\right)\right|<\delta, \\
& \left|\left(F_{\alpha}^{k}\right)_{i}\left(x_{1}, \ldots, x_{i-1}, \gamma_{1}, x_{1+i}, \ldots, x_{k}\right)-f\left(\gamma_{1}\right)\right|<\delta .
\end{aligned}
$$

Next, let us consider the translations $t_{v}, t_{w}$ according to the vectors $v, w$ given by

$$
v=\left(-\frac{\beta_{0}+\beta_{1}}{2}, \ldots,-\frac{\beta_{0}+\beta_{1}}{2}\right), \quad w=\left(-\frac{\gamma_{0}+\gamma_{1}}{2}, \ldots,-\frac{\gamma_{0}+\gamma_{1}}{2}\right),
$$

respectively, and the maps $h_{0}, h_{1}$ defined by

$$
h_{0}\left(x_{1}, \ldots, x_{k}\right)=\frac{2}{\beta_{1}-\beta_{0}}\left(x_{1}, \ldots, x_{k}\right), \quad h_{1}\left(x_{1}, \ldots, x_{k}\right)=\frac{2}{\gamma_{1}-\gamma_{0}}\left(x_{1}, \ldots, x_{k}\right) .
$$

We claim that the $h$-cubes $N_{0}, N_{1}$ with

- $u\left(N_{0}\right)=u\left(N_{1}\right)=k$ and $s\left(N_{0}\right)=s\left(N_{1}\right)=0$,

- $c_{N_{0}}=h_{0} \circ t_{v}, c_{N_{1}}=h_{1} \circ t_{w}$, 
satisfy the covering relations

$$
N_{i} \stackrel{F_{\alpha}^{k}}{\Longrightarrow} N_{j}
$$

for all $i, j=0,1$. Note that $N_{c}^{+}=\emptyset$ and $N_{c}^{-}=\partial J_{k}$ for $N=N_{i}, i=0,1$.

We first prove the relation

$$
N_{0} \stackrel{F_{\alpha}^{k}}{\Longrightarrow} N_{0}
$$

To this end, we take the linear map $A(x)=2 x$ and define the homotopy $H:[0,1] \times J_{k} \longrightarrow \mathbb{R}^{k}$ by

$$
H(t, x)=t A(x)+(1-t)\left(c_{N_{0}} \circ F_{\alpha}^{k} \circ c_{N_{0}}^{-1}\right)(x) .
$$

Clearly, $H(0, \cdot)=c_{N_{0}} \circ F_{\alpha}^{k} \circ c_{N_{0}}^{-1}$ and $H(1, \cdot)=A$. Next we prove that

$$
H\left(t, \partial J_{k}\right) \cap J_{k}=\emptyset .
$$

Indeed, take a point $x \in \partial J_{k}$. For this point, we can ensure that there is an index $i \in\{1, \ldots, k\}$ so that $x_{i}$ is equal either to -1 or to 1 . Assume that we are in the first case (the proof in the other case is completely analogous). By the definition of $c_{N_{0}}$, we deduce that

$$
c_{N_{0}}^{-1}(x)=\left(y_{1}, \ldots, y_{i-1}, \beta_{0}, y_{i+1}, \ldots, y_{k}\right),
$$

with $\left(y_{1}, \ldots, y_{i-1}, y_{i+1}, \ldots, y_{k}\right) \in\left[\beta_{0}, \beta_{1}\right]^{k-1}$. Now, using condition (4.1), it follows that

$$
\left|\left(F_{\alpha}^{k}\right)_{i}\left(y_{1}, \ldots, y_{i-1}, \beta_{0}, y_{i+1}, \ldots, y_{k}\right)-f\left(\beta_{0}\right)\right|<\delta .
$$

Therefore, as $f$ is $\delta$-strictly turbulent, we obtain that

$$
\left(F_{\alpha}^{k}\right)_{i}\left(y_{1}, \ldots, y_{i-1}, \beta_{0}, y_{i+1}, \ldots, y_{k}\right)<\beta_{0} .
$$

Finally, by the definition of $c_{N_{0}}$, we arrive at

$$
\left(c_{N_{0}} \circ F_{\alpha}^{k}\right)_{i}\left(y_{1}, \ldots, y_{i-1}, \beta_{0}, y_{i+1}, \ldots, y_{k}\right)<-1 .
$$

Bringing all the information together, and using that $(A x)_{i}=2 x_{i}=-2$, it is clear that

$$
t(A x)_{i}+(1-t)\left(c_{N_{0}} \circ F_{\alpha}^{k} \circ c_{N_{0}}^{-1}(x)\right)_{i}<-1
$$

for all $t \in[0,1]$, and therefore $H(t, x) \notin J_{k}$. The proof of (4.5) is now complete. The same reasoning applies to checking the remaining covering relations. We just note that for the relations

$$
N_{1} \stackrel{F_{\alpha}^{k}}{\Longrightarrow} N_{i}
$$

with $i=0,1$, the choice for the linear map is $A(x)=-2 x$.

Remark 4.1. If some iteration $f^{m}$ is $\delta$-strictly turbulent, then the conclusion of Theorem 4.2 holds, replacing $F_{\alpha}^{k}$ by $F_{\alpha}^{k m}$. 
Remark 4.2. The requirement of an upper bound in the range of $\alpha$ imposed in Theorem 4.2 is necessary. For values of $\alpha$ close to $1,(2.1)$ has a globally stable positive equilibrium (see $[13,20]$ and their references). Actually, for $k=1, \alpha$ is sometimes used as a control parameter so that increasing its value becomes an effective tool for chaos control [22].

To ensure the existence of chaotic dynamics in (2.1) for a particular choice of $\alpha$ when $f$ is $\delta$-strictly turbulent, we have to verify conditions (4.1)-(4.4). We illustrate this fact with an example.

Example 4.1. Consider the system

$$
x_{n}=\alpha x_{n-1}+(1-\alpha) f\left(x_{n-2}\right),
$$

where $f(x)=x \exp (4-x)$. A direct computation allows us to check that $f^{2}$ is 2.4-strictly turbulent with parameters $3<5.75<6.25<11$. In Appendix A, we show that conditions (4.1)-(4.4) hold for $f^{2}$ with $\alpha_{0}=0.001$.

It is worth pointing out some biological implications of our results for (4.6). Specifically, there are two disjoint regions, namely

$$
N_{0}=[3,5.75]^{2}, \quad N_{1}=[6.25,11]^{2},
$$

with the "coin-tossing" property for $F_{\alpha}^{4}$. For instance, if we take the bi-infinite sequence

$$
(\ldots, 1,0,1,0,0,1,0,0,0,1,0,0,0,0,1, \ldots)
$$

(assume that the first 1 corresponds to the index 0 in the sequence), we can choose a point $x$ so that

$$
x \in N_{1}, F_{\alpha}^{4}(x) \in N_{0}, F_{\alpha}^{8}(x) \in N_{1}, F_{\alpha}^{12}(x) \in N_{0}, F_{\alpha}^{16}(x) \in N_{0}, F_{\alpha}^{20}(x) \in N_{1}, \ldots
$$

Moreover, if the sequence is periodic, we can take a periodic point following the "itinerary" of the sequence. As a direct consequence of Proposition 3.5, we can also estimate the sensitive dependence. Indeed, for $\varepsilon>0$, clearly

$$
S_{\varepsilon} \leq\left\lceil\frac{(5.75-3)^{2}}{\varepsilon^{2}}\right\rceil:=B .
$$

Hence, there are two points $x_{0}, y_{0} \in[3,5.75]^{2}$ such that $\left\|x_{0}-y_{0}\right\|<\varepsilon$ and, for some number $j \in\left\{1, \ldots, N^{*}\right\}$, we have that $F^{4 j}\left(x_{0}\right) \in[3,5.75]^{2}$ and $F^{4 j}\left(y_{0}\right) \in[6.25,11]^{2}$. Therefore,

$$
\left\|F^{4 j}\left(x_{0}\right)-F^{4 j}\left(y_{0}\right)\right\| \geq 0.5 \text {. }
$$

We emphasize that, for any given value of $\varepsilon>0$, we can compute an explicit bound $\left\lceil\frac{\ln B}{\ln 2}\right\rceil+1$ for $N^{*}$.

Next we study the LPA model (2.3), whose solutions are the orbits of the map $G: \mathbb{R}^{3} \rightarrow \mathbb{R}^{3}$ defined by

$$
G(L, P, A)=\left(b A \exp \left(-c_{e l} L-c_{e a} A\right),\left(1-\mu_{l}\right) L, P \exp \left(-c_{p a} A\right)+\left(1-\mu_{a}\right) A\right) .
$$


Clearly, for $c_{e l}=\mu_{l}=c_{p a}=0$ and $\mu_{a}=1$, system (2.3) becomes Clark's model with $\alpha=0$, $k=3$, and $f(x)=b x e^{-c_{e a} x}$. Thus, the proof of Theorem 4.2 can be easily adapted to deal with (2.3). Since there are values of $b$ and $c_{e a}$ so that $f^{2}$ is $\delta$-strictly turbulent, the following result establishes chaos for the LPA model.

Theorem 4.3. Assume that $f^{2}$ is $\delta$-strictly turbulent with parameters $\beta_{0}<\beta_{1}<\gamma_{0}<\gamma_{1}$ and $\delta>0$. There exists $\sigma>0$ so that if $0 \leq c_{e l}, \mu_{l}, c_{p a} \leq \sigma$ and $1-\sigma \leq \mu_{a} \leq 1$, then $G^{6}$ induces chaotic dynamics on two symbols relative to $N_{0}=\left[\beta_{0}, \beta_{1}\right]^{3}$ and $N_{1}=\left[\gamma_{0}, \gamma_{1}\right]^{3}$, where $G$ is the map defined in (4.7).

We observe that, reasoning in a similar way as in the previous example, it is possible to estimate properties of sensitive dependence on the initial conditions for particular cases of system (2.3).

To the best of the authors' knowledge, Theorem 4.3 is the first analytic result of chaotic dynamics for the LPA model in the interior of the nonnegative cone $\mathbb{R}_{+}^{3}$. It is worth mentioning that in [10, Theorem 2], Cushing proves analytically the existence of fully synchronous orbits with chaotic behavior when $\mu_{a}=1$.

5. Chaotic dynamics in system (2.2). In this section we apply the results of section 3 to the nonlinear Leslie population model (2.2). In contrast to the previous section, in this case we find a chaotic regime where the map of the system has one stable direction and one unstable (in the sense of Definition 3.2). Associated with (2.2), we define the map in $\mathbb{R}^{2}$,

$$
F(x, y)=\left(\left(a_{1} x+a_{2} y\right) \exp \left(-\lambda_{1} x-\lambda_{2} y\right), b x\right),
$$

where all the parameters are strictly positive and $b \in] 0,1]$. Our main result in this section is the following.

Theorem 5.1. Assume that $f(x)=a_{1} x \exp \left(-\lambda_{1} x\right)$ satisfies that $f^{2}$ is $\delta$-strictly turbulent with parameters $\alpha_{0}<\alpha_{1}<\beta_{0}<\beta_{1}$ and $\delta>0$. Suppose that there is $r>0$ so that the following inequalities are fulfilled:

$$
\begin{aligned}
\frac{b}{e}\left(\frac{a_{1}}{\lambda_{1}}+\frac{a_{2}}{\lambda_{2}}\right) & <r, \\
\frac{-a_{1}}{e}\left(e^{-\lambda_{2} r}-1\right) & <\ln \left(\frac{\alpha_{0}-a_{1} a_{2} r-a_{2} b \beta_{1}}{\alpha_{0}-\delta}\right), \\
\lambda_{2} r+\lambda_{2} b \beta_{1}+\lambda_{1} a_{2} r & <\ln \left(\frac{\beta_{1}+\delta}{\beta_{1}}\right) .
\end{aligned}
$$

Then $F^{2}$ induces chaotic dynamics on two symbols relative to $N_{0}=\left[\alpha_{0}, \alpha_{1}\right] \times[0, r]$ and $N_{1}=\left[\beta_{0}, \beta_{1}\right] \times[0, r]$.

Proof. It is easy to check that $N_{0}$ and $N_{1}$ are $h$-sets, with

- $u\left(N_{0}\right)=u\left(N_{1}\right)=1$ (x-direction) and $s\left(N_{0}\right)=s\left(N_{1}\right)=1$ (y-direction),

- $c_{N_{0}}=h_{0} \circ t_{v}$ and $c_{N_{1}}=h_{1} \circ t_{w}$,

where $t_{v}$ and $t_{w}$ are the translations according to the vectors

$$
v=\left(\frac{-\left(\alpha_{0}+\alpha_{1}\right)}{2}, \frac{-r}{2}\right), \quad w=\left(\frac{-\left(\beta_{0}+\beta_{1}\right)}{2}, \frac{-r}{2}\right)
$$


respectively, and

$$
h_{0}(x, y)=\left(\frac{2 x}{\alpha_{1}-\alpha_{0}}, \frac{2 y}{r}\right), \quad h_{1}(x, y)=\left(\frac{2 x}{\beta_{1}-\beta_{0}}, \frac{2 y}{r}\right) .
$$

We have to demonstrate that

$$
N_{i} \stackrel{F^{2}}{\Longrightarrow} N_{j}
$$

for $i, j=0,1$. We give the proof only for the case $i=j=0$. Indeed, consider the homotopy

$$
H(t,(x, y))=t\left(c_{N_{0}} \circ F^{2} \circ c_{N_{0}}^{-1}\right)(x, y)+(1-t) A(x, y),
$$

where $A(x, y)=(2 x, 0)$.

Define $f(x)=a_{1} x e^{-\lambda_{1} x}$. After some elementary computations (see Appendix B), we arrive at the inequalities

$$
\begin{aligned}
& F_{1}^{2}(x, y) \leq f^{2}(x)\left(\exp \left(-a_{1} / e\right)\right)^{\exp \left(-\lambda_{2} y\right)-1}+a_{1} a_{2} y+a_{2} b x, \\
& F_{1}^{2}(x, y) \geq f^{2}(x) \exp \left(-\lambda_{2} b x\right) \exp \left(-\lambda_{1} a_{2} y\right) \exp \left(-\lambda_{2} y\right), \\
& F_{2}^{2}(x, y) \leq b\left(\frac{a_{1}}{\lambda_{1} e}+\frac{a_{2}}{\lambda_{2} e}\right) .
\end{aligned}
$$

Now, since $b>0$, we deduce from (5.1) that, for all $(x, y) \in N_{0}$,

$$
0<F_{2}^{2}(x, y)<r .
$$

Analogously, as $f^{2}\left(\alpha_{0}\right)<\alpha_{0}-\delta$, we obtain that

$$
F_{1}^{2}\left(\alpha_{0}, y\right) \leq\left(\alpha_{0}-\delta\right)\left(\exp \left(-a_{1} / e\right)\right)^{\exp \left(-\lambda_{2} r\right)-1}+a_{1} a_{2} r+a_{2} b \alpha_{0}
$$

for all $y \in[0, r]$. By using (5.2) together with the inequality $\alpha_{0}<\beta_{1}$, we get from expression (5.5) that, for all $y \in[0, r]$,

$$
F_{1}^{2}\left(\alpha_{0}, y\right)<\alpha_{0}
$$

Reasoning in the same way with condition (5.3), it may be concluded that

$$
F_{1}^{2}\left(\alpha_{1}, y\right)>\alpha_{1}
$$

for all $y \in[0, r]$.

From inequalities (5.4), (5.6), and (5.7), it follows that

$$
\begin{aligned}
c_{N_{0}} \circ F^{2} \circ c_{N_{0}}^{-1}(\{-1\} \times[-1,1]) & \subset\{(x, y): x<-1\}, \\
c_{N_{0}} \circ F^{2} \circ c_{N_{0}}^{-1}(\{1\} \times[-1,1]) & \subset\{(x, y): x>1\}, \\
c_{N_{0}} \circ F^{2} \circ c_{N_{0}}^{-1}\left([-1,1]^{2}\right) & \subset\{(x, y):-1<y<1\} .
\end{aligned}
$$


These properties, together with the expression of $A$, lead to the desired conclusion,

$$
\begin{array}{r}
H([0,1],\{-1,1\} \times[-1,1]) \cap[-1,1]^{2}=\emptyset, \\
H\left([0,1],[-1,1]^{2}\right) \cap([-1,1] \times\{-1,1\})=\emptyset .
\end{array}
$$

The same steps lead to the covering relation

$$
N_{0} \stackrel{F^{2}}{\Longrightarrow} N_{1}
$$

For the relations

$$
N_{1} \stackrel{F^{2}}{\Longrightarrow} N_{i}
$$

with $i=0,1$, the most significant change consists of taking the linear map $A(x, y)=$ $(-2 x, 0)$.

Now we apply Theorem 5.1 in a particular example. Take $f(x)=x e^{4-x}\left(a_{1}=e^{4}, \lambda_{1}=1\right)$. As claimed in section $4, f^{2}$ is 2.4 -strictly turbulent with parameters $3<5.75<6.25<11$. Straightforward computations show that conditions (5.1)-(5.3) hold for $r=0.065, b=0.003$, $a_{2}=0.1$, and $\lambda_{2}=1$.

In general, to use Theorem 5.1, the parameters $b$ and $r$ must be close to zero. Nevertheless, for values of $b$ near 1 , system (2.2) also displays chaotic dynamics; indeed, for $a_{1}=\lambda_{1}=0$ and $b=1$, we again recover Clark's model (2.1) with $\alpha=0$. Consequently, arguing as in the proof of Theorem 4.2, we are able to guarantee the existence of chaos for $(2.2)$ when $\left(a_{1}, \lambda_{1}, b\right)$ belongs to a neighborhood of $(0,0,1)$. It is important to observe that, from a mathematical point of view, the chaotic regimes are different because in Theorem 5.1 we have one stable direction and one unstable direction, while in Theorem 4.2 both directions are unstable.

6. Discussion. We have proved analytic criteria for chaotic dynamics for some well-known structured population models, and we have given further insight into such relevant features of chaos as the sensitive dependence on initial conditions. We list the main advantages of our results:

- Explicit ranges of parameters where models (2.1), (2.2), and (2.3) exhibit chaotic behavior: A key property from a biological point of view is the robustness of our results under small continuous perturbations. This aspect is especially important in modeling, because the coefficients involved are usually approximations derived from experimental data, so small errors should be taken into account.

- Regions of initial data with chaotic behavior: We identify explicit regions of the phase space, namely $N_{0}$ and $N_{1}$, where chaotic behavior occurs. Determining these regions is useful for analyzing the influence of the chaotic regime on the system since, in some situations, a chaotic attractor may coexist with other dynamics, such as a locally stable periodic point.

- Estimates of the sensitive dependence: The strategy followed in our proofs allows us to get quantitative results about sensitive dependence on initial conditions in an easy way; see Proposition 3.5 and Example 4.1. 
In the literature there are several papers dealing with the existence of chaotic dynamics for structured population models. However, most of them generally rely on numerical evidence obtained from computer simulations, particularly on the computation of the dominant Lyapunov exponent (see, for instance, [16] for Clark's equation, and [11] for the LPA model).

For the nonlinear Leslie model (2.2), our results complement Theorem 1.2 in [33], which, based on the results of [34], ensures the existence of a chaotic attractor for a set of parameters with positive Lebesgue measure; however, compared with our approach, determining such a set can be much more involved. We note that our results provide criteria only for the existence of invariant sets with chaotic dynamics, not attractors. In particular, this complex behavior might not be observed in numerical simulations of the models. We point out that Theorem 1.2 in [33] establishes stronger attraction and stochastic properties.

Appendix A. Computations for Example 4.1. This appendix contains some auxiliary calculations for Example 4.1.

For $F_{\alpha}\left(x_{1}, x_{2}\right)=\left(x_{2}, \alpha x_{2}+(1-\alpha) f\left(x_{1}\right)\right)$, the expression of $F_{\alpha}^{4}=\left(\left(F_{\alpha}^{4}\right)_{1},\left(F_{\alpha}^{4}\right)_{2}\right)$ is given by

$$
\begin{aligned}
\left(F_{\alpha}^{4}\right)_{1}\left(x_{1}, x_{2}\right)= & \alpha^{3} x_{2}+\alpha^{2}(1-\alpha) f\left(x_{1}\right)+\alpha(1-\alpha) f\left(x_{2}\right) \\
& +(1-\alpha) f\left(\alpha x_{2}+(1-\alpha) f\left(x_{1}\right)\right) \\
\left(F_{\alpha}^{4}\right)_{2}\left(x_{1}, x_{2}\right)= & \alpha^{4} x_{2}+\alpha^{3}(1-\alpha) f\left(x_{1}\right)+\alpha^{2}(1-\alpha) f\left(x_{2}\right) \\
& +\alpha(1-\alpha) f\left(\alpha x_{2}+(1-\alpha) f\left(x_{1}\right)\right) \\
& +(1-\alpha) f\left(\alpha^{2} x_{2}+\alpha(1-\alpha) f\left(x_{1}\right)+(1-\alpha) f\left(x_{2}\right)\right) .
\end{aligned}
$$

Next we notice that, in $[0,+\infty$ [, function $f(x)=x \exp (4-x)$ is bounded by $\exp (3)$ and is Lipschitz-continuous with Lipschitz-constant $\exp (4)$. Using these properties in a direct way, we obtain that, for all $x_{2} \in[3,5.75] \cup[6.25,11]$,

$$
\begin{aligned}
\left|\left(F_{\alpha}^{4}\right)\left(x_{1}, x_{2}\right)_{1}-f^{2}\left(x_{1}\right)\right| \leq & 11 \alpha^{3}+\exp (3) \alpha^{2}(1-\alpha)+\exp (3) \alpha(1-\alpha) \\
& +\exp (4)(11 \alpha+\exp (3) \alpha)+\alpha \exp (3):=\varphi(\alpha),
\end{aligned}
$$

and, for all $x_{1} \in[3,5.75] \cup[6.25,11]$,

$$
\begin{aligned}
\left|\left(F_{\alpha}^{4}\right)\left(x_{1}, x_{2}\right)_{2}-f^{2}\left(x_{2}\right)\right| \leq & 11 \alpha^{4}+\alpha^{3}(1-\alpha) \exp (3)+\alpha^{2}(1-\alpha) \exp (3)+\alpha(1-\alpha) \exp (3) \\
& +11 \exp (4) \alpha^{2}+\exp (7) \alpha(1-\alpha)+\exp (7) \alpha+\exp (3) \alpha:=\psi(\alpha) .
\end{aligned}
$$

Now, since $\varphi$ and $\psi$ are polynomials, it is elementary to check that $\varphi(\alpha)<2.4$ and $\psi(\alpha)<2.4$ for all $\alpha<\alpha_{0}=0.001$ (see Figure 1).

Appendix B. Auxiliary calculations for the proof of Theorem 5.1. In this appendix, we provide some expressions and preliminary calculations for the proof of Theorem 5.1.

Consider the map

$$
F(x, y)=\left(\left(a_{1} x+a_{2} y\right) \exp \left(-\lambda_{1} x-\lambda_{2} y\right), b x\right) .
$$




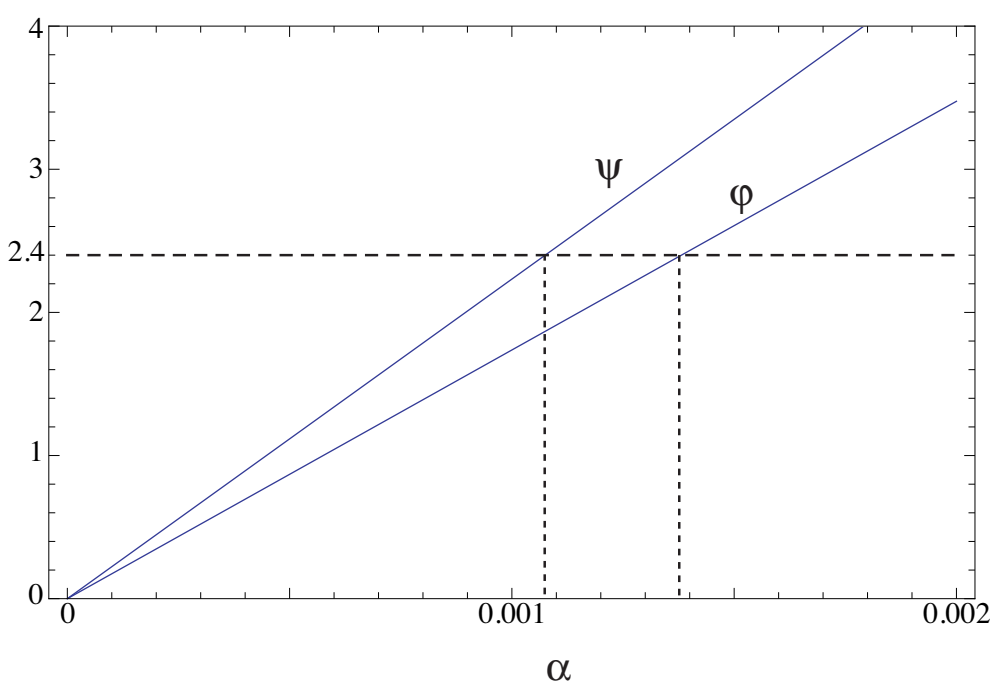

Figure 1. Representation of functions $\varphi(\alpha)$ and $\psi(\alpha)$ for $0 \leq \alpha \leq 0.002$.

The first component of $F^{2}$ has the following expression:

$$
\begin{aligned}
F_{1}^{2}(x, y)=e^{-\lambda_{1} a_{1} x \exp \left(-\lambda_{1} x-\lambda_{2} y\right)} e^{-\lambda_{2} b x} e^{-\lambda_{1} a_{2} y \exp \left(-\lambda_{1} x-\lambda_{2} y\right)} & {\left[a_{1}^{2} x e^{-\lambda_{1} x-\lambda_{2} y}\right.} \\
& \left.+a_{1} a_{2} y e^{-\lambda_{1} x-\lambda_{2} y}+a_{2} b x\right] .
\end{aligned}
$$

We easily deduce that, for $x \geq 0$ and $y \geq 0$,

$$
F_{1}^{2}(x, y) \leq a_{1}^{2} x e^{-\lambda_{1} x} e^{-\lambda_{1} a_{1} x \exp \left(-\lambda_{1} x\right)}\left(e^{-\lambda_{1} a_{1} x \exp \left(-\lambda_{1} x\right)}\right)^{e^{-\lambda_{2} y}-1}+a_{1} a_{2} y+a_{2} b x .
$$

Now, using that $f(x)=a_{1} x e^{-\lambda_{1} x} \leq \frac{a_{1}}{\lambda_{1} e}$, we arrive at

$$
\begin{aligned}
F_{1}^{2}(x, y) & \leq a_{1}^{2} x e^{-\lambda_{1} x} e^{-\lambda_{1} a_{1} x \exp \left(-\lambda_{1} x\right)}\left(e^{\frac{-a_{1}}{e}}\right)^{e^{-\lambda_{2} y}-1}+a_{1} a_{2} y+a_{2} b x \\
& =f^{2}(x)\left(e^{\frac{-a_{1}}{e}}\right)^{e^{-\lambda_{2} y}-1}+a_{1} a_{2} y+a_{2} b x .
\end{aligned}
$$

On the other hand,

$$
\begin{aligned}
F_{1}^{2}(x, y) & \geq e^{-\lambda_{1} a_{1} x \exp \left(-\lambda_{1} x-\lambda_{2} y\right)} e^{-\lambda_{2} b x} e^{-\lambda_{1} a_{2} y \exp \left(-\lambda_{1} x-\lambda_{2} y\right)} a_{1}^{2} x e^{-\lambda_{1} x-\lambda_{2} y} \\
& \geq a_{1}^{2} x e^{-\lambda_{1} x} e^{-\lambda_{1} a_{1} x \exp \left(-\lambda_{1} x\right)} e^{-\lambda_{2} b x} e^{-\lambda_{1} a_{2} y} e^{-\lambda_{2} y} \\
& =f^{2}(x) e^{-\lambda_{2} b x} e^{-\lambda_{1} a_{2} y} e^{-\lambda_{2} y} .
\end{aligned}
$$

For the second component, using that $f(x)=a_{1} x e^{-\lambda_{1} x} \leq \frac{a_{1}}{\lambda_{1} e}$, we get

$$
F_{2}^{2}(x, y)=b\left(a_{1} x+a_{2} y\right) e^{-\lambda_{1} x-\lambda_{2} y} \leq b\left(\frac{a_{1}}{\lambda_{1} e}+\frac{a_{2}}{\lambda_{2} e}\right) .
$$


Acknowledgments. We thank an anonymous referee for his/her useful comments. The second author thanks the Departamento de Matemática Aplicada II of the University of Vigo (Spain) for its kind hospitality.

\section{REFERENCES}

[1] B. Aulbach And B. Kieninger, On three definitions of chaos, Nonlinear Dyn. Syst. Theory, 1 (2001), pp. 23-37.

[2] L. S. Block And W. A. Coppel, Dynamics in One Dimension, Lectures Notes in Math. 1513, Springer, Berlin, 1992.

[3] L. W. Botsford, Further analysis of Clark's delayed recruitment model, Bull. Math. Biol., 54 (1992), pp. $275-293$.

[4] H. Caswell, Matrix Population Models: Construction, Analysis and Interpretation, 2nd ed., Sinauer Associates, Sunderland, MA, 2001.

[5] C. W. Clark, A delayed recruitment model of population dynamics with an application to baleen whale populations, J. Math. Biol., 3 (1976), pp. 381-391.

[6] C. W. Clark, Mathematical Bioeconomics: The Optimal Management of Renewable Resources, 2nd ed., John Wiley \& Sons, Hoboken, NJ, 1990.

[7] R. F. Costantino, J. M. Cushing, B. Dennis, and R. A. Desharnais, Experimentally induced transitions in the dynamic behaviour of insect populations, Nature, 375 (1995), pp. 227-230.

[8] R. F. Costantino, R. A. Desharnais, J. M. Cushing, and B. Dennis, Chaotic dynamics in an insect population, Science, 275 (1997), pp. 389-391.

[9] J. M. Cushing, An Introduction to Structured Population Dynamics, CBMS-NSF Regional Conf. Ser. in Appl. Math. 71, SIAM, Philadelphia, 1998.

[10] J. M. Cushing, Cycle chains and the LPA model, J. Differ. Equations Appl., 9 (2003), pp. 655-670.

[11] J. M. Cushing, R. F. Costantino, B. Dennis, R. A. Desharnais, and S. M. Henson, Chaos in Ecology: Experimental Nonlinear Dynamics, Theoret. Ecol. Ser., Academic Press, New York, 2003.

[12] J. M. Cushing, The LPA model, in Fields Inst. Commun. 42, AMS, Providence, RI, 2004, pp. 29-55.

[13] H. A. El-Morshedy And E. Liz, Globally attracting fixed points in higher order discrete population models, J. Math. Biol., 53 (2006), pp. 365-384.

[14] J. Guckenheimer, G. Oster, And A. Ipaktchi, The dynamics of density dependent population models, J. Math. Biol., 4 (1977), pp. 101-147.

[15] A. Hastings, C. L. Hom, S. P. Ellner, P. Turchin, and H. C. J. Godfray, Chaos in ecology: Is mother nature a strange attractor?, Ann. Rev. Ecol. Syst., 24 (1993), pp. 1-33.

[16] K. Higgins, A. Hastings, And L. W. Botsford, Density dependence and age structure: Nonlinear dynamics and population behavior, Amer. Nat., 149 (1997), pp. 247-269.

[17] J. Kennedy, S. Koçak, And J. A. Yorke, A chaos lemma, Amer. Math. Monthly, 108 (2001), pp. 411423.

[18] U. Kirchgraber And D. Stoffer, On the definition of chaos, ZAMM Z. Angew. Math. Mech., 69 (1989), pp. 175-185.

[19] S. A. Levin and C. P. Goodyear, Analysis of an age-structured fishery model, J. Math. Biol., 9 (1980), pp. 245-274.

[20] E. Liz, Global stability and bifurcations in a delayed discrete population model, Int. J. Qual. Theory Differential Equations Appl., 3 (2009), pp. 66-80.

[21] E. Liz, Complex dynamics of survival and extinction in simple population models with harvesting, Theor. Ecol., 3 (2010), pp. 209-221.

[22] E. Liz AND D. Franco, Global stabilization of fixed points using predictive control, Chaos, 20 (2010), 023124.

[23] E. Liz And A. Ruiz-Herrera, The hydra effect, bubbles, and chaos in a simple discrete population model with constant effort harvesting, J. Math. Biol., (2012), to appear; available online as 10.1007/s00285011-0489-2.

[24] R. M. MAY, Biological populations with nonoverlapping generations: Stable points, stable cycles, and 
chaos, Science, 186 (1974), pp. 645-647.

[25] R. M. MAY, Simple mathematical models with very complicated dynamics, Nature, 261 (1976), pp. 459467.

[26] R. M. MAY, Mathematical models in whaling and fisheries management, in Some Mathematical Questions in Biology, G. F. Oster, ed., AMS, Providence, RI, 1980, pp. 1-64.

[27] A. Medio, M. Pireddu, And F. Zanolin, Chaotic dynamics for maps in one and two dimensions: A geometrical method and applications to economics, Internat. J. Bifur. Chaos Appl. Sci. Engrg., 19 (2009), pp. 3283-3309.

[28] S. Smale, Finding a horseshoe on the beaches of Rio, Math. Intelligencer, 20 (1998), pp. 39-44.

[29] H. R. Thieme, Mathematics in Population Biology, Princeton University Press, Princeton, NJ, 2003.

[30] H. Thunberg, Periodicity versus chaos in one-dimensional dynamics, SIAM Rev., 43 (2001), pp. 3-30.

[31] S. Tuljapurkar and H. Caswell, eds., Structured-Population Models in Marine, Terrestrial, and Freshwater Systems, Population Community Biol. Ser. 18, Chapman \& Hall, New York, 1997.

[32] I. Ugarcovici AND H. Weiss, Chaotic dynamics of a nonlinear density dependent population model, Nonlinearity, 17 (2004), pp. 1689-1711.

[33] I. Ugarcovici AND H. WeIss, Chaotic attractors and physical measures for some density dependent Leslie population models, Nonlinearity, 20 (2007), pp. 2897-2906.

[34] Q. Wang And L.-S. Young, Strange attractors with one direction of instability, Commun. Math. Phys., 218 (2001), pp. 1-97.

[35] A. WiKAn AND E. MJøLhus, Overcompensatory recruitment and generation delay in discrete agestructured population models, J. Math. Biol., 35 (1996), pp. 195-239.

[36] A. A. Yakubu, N. Li, J. M. Conrad, and M. L. Zeeman, Constant proportion harvest policies: Dynamic implications in the Pacific halibut and Atlantic cod fisheries, Math. Biosci., 232 (2011), pp. 66-77.

[37] P. Zgliczyński and M. Gidea, Covering relations for multidimensional dynamical systems, J. Differential Equations, 202 (2004), pp. 32-58.

[38] P. ZGLICZYŃSKi, On periodic points for systems of weakly coupled 1-dim maps, Nonlinear Anal., 46 (2001), pp. 1039-1062. 